\title{
Mapping multiple QTL using linkage disequilibrium and linkage analysis information and multitrait data
}

\author{
Theo H.E. Meuwissen ${ }^{\mathrm{a} *}$, Mike E. GodDARD ${ }^{\mathrm{b}}$ \\ ${ }^{\text {a }}$ Centre for Integrative Genetics (Cigene), Institute of Animal Science, Agricultural University \\ of Norway, Box 5025, Ås, Norway \\ ${ }^{\mathrm{b}}$ Institute of Land and Food Resources, University of Melbourne, and Victorian Institute \\ of Animal Science, Attwood, Australia
}

(Received 17 February 2003; accepted 10 November 2003)

\begin{abstract}
A multi-locus QTL mapping method is presented, which combines linkage and linkage disequilibrium (LD) information and uses multitrait data. The method assumed a putative QTL at the midpoint of each marker bracket. Whether the putative QTL had an effect or not was sampled using Markov chain Monte Carlo (MCMC) methods. The method was tested in dairy cattle data on chromosome 14 where the DGAT1 gene was known to be segregating. The DGAT1 gene was mapped to a region of $0.04 \mathrm{cM}$, and the effects of the gene were accurately estimated. The fitting of multiple QTL gave a much sharper indication of the QTL position than a single QTL model using multitrait data, probably because the multi-locus QTL mapping reduced the carry over effect of the large DGAT1 gene to adjacent putative QTL positions. This suggests that the method could detect secondary QTL that would, in single point analyses, remain hidden under the broad peak of the dominant QTL. However, no indications for a second QTL affecting dairy traits were found on chromosome 14 .
\end{abstract}

QTL mapping / linkage analysis / linkage disequilibrium mapping / multitrait analysis / multi-locus mapping

\section{INTRODUCTION}

Quantitative trait loci (QTL) mapping methods that fit a single QTL to the data can be biased by the presence of other QTL, especially if they are close to the putative QTL position. In extreme situations, two linked QTL can cancel each others effects, and none of the QTL is detected. In other situations a 'ghost' QTL is found in between two real QTL [18]. This problem may be remedied by fitting two or more QTL simultaneously. The question arises how

*Corresponding author: theo.meuwissen@iha.nlh.no 
many QTL should be fitted. This is a model selection problem, i.e. which and how many QTL should the model contain. Many methods for model selection have been described in the literature $[5,26]$, of which perhaps Akaikes Information Criterion [2], which corrects the model likelihood for the number of parameters fitted, is the most well known. In Bayesian statistics, model selection may be part of the statistical inference, i.e. prediction of the posterior probability of the alternative models is part of the analysis. More importantly, the posterior probability of having a QTL at map position, say, $10 \mathrm{cM}$ is obtained by integrating over all the possible models, i.e. the position estimate accounts for the model uncertainty. Sillanpaa and Arjas [24] and Bink et al. [4] used Bayesian model selection techniques to estimate the number of QTL and their positions simultaneously.

The power to detect QTL and the accuracy of estimating QTL positions may be improved by using the information from all traits simultaneously (i.e. multitrait QTL mapping), instead of using several single trait analyses [29]. The assumption here is that the QTL has pleiotropic effects on the traits included in the multitrait analysis, and that a multitrait analysis combines the information from each of the traits. Multitrait QTL mapping is especially beneficial when the pleiotropic effects of the QTL differ substantially from the most frequently observed effects of the environments and background genes, which is reflected by the environmental and background genetic correlations. However, multitrait models require an increased number of parameters estimated, which may diminish their usefulness in small data sets. Especially, in the case of QTL mapping by variance components where the infinite alleles model is used to model the QTL effects $[12,14]$, this requires the estimation of

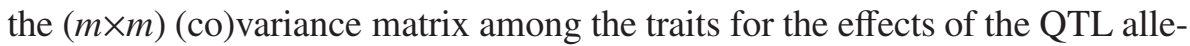
les [17], where $m=$ number of traits, i.e. the number of (co)variance estimates increases quadratically with the number of traits. Mapping by variance components will also be used here, but we adopt the suggestion of Goddard [11] that the correlations among QTL effects at a single gene are either +1 or -1 . This assumption is always valid if there are only two alleles segregating at the QTL and may be reasonable for more alleles. This approach implies that the number of parameters for the QTL effect increases linearly, not quadratically, with the number of traits.

Another way to improve the power and precision of QTL mapping is to make use of linkage disequilibrium (LD) information, which implies that the information of historical recombinations is used [15]. Linkage disequilibria can be found over large distances [7], and thus pure LD analysis can easily result in the detection of false positives. It is therefore important to detected LD 
that are due to close linkage, and the combined use of linkage disequilibrium and linkage analysis information will avoid spurious long distance associations leading to false likelihood peaks, because the linkage analysis information will not confirm such spurious associations [19,21,22]. Variance component mapping can account for the LD information by simply relaxing the assumption that the founder QTL alleles in the infinite alleles model are unrelated. Identityby-descent (IBD) probabilities between the founder QTL alleles are estimated from the similarities between the surrounding marker-haplotypes [20].

The aim of this paper is to combine the above approaches, that improve the power and precision of QTL mapping, into one method. A QTL mapping by variance components method will be presented that performs a Bayesian integration over zero, one, two, and more QTL models, and uses the information from multiple traits, LD and linkage analysis simultaneously to map the QTL as accurate as possible. The presented method will be applied in a dairy data set of chromosome 14, where the DGAT1 gene has been found previously [13], and thus the exact position of the QTL is known.

\section{METHODS}

\subsection{The multi-trait multi-QTL model}

The vector of $m$ phenotypic records of animal $i, \mathbf{y}_{i}$, is modeled by:

$$
\mathbf{y}_{i}=\mathbf{X}_{i} \mathbf{b}+\mathbf{u}_{i}+\Sigma_{j}\left(\mathrm{q}_{i j 1}+\mathrm{q}_{i j 2}\right) \mathbf{v}_{j}+\mathbf{e}_{i}
$$

where $\mathbf{y}_{i}$ here is the $(m \times 1)$ vector of daughter yield deviation (DYD) of sire $i$; $\mathbf{X}_{i} \mathbf{b}$ denotes the $(m \times 1)$ vector of (non-genetic) fixed effect corrections for the traits of animal $i$; $\mathbf{u}_{i}=(m \times 1)$ vector of effects of the background genes (polygenic effect) on each of the traits; $\mathbf{e}_{i}=(m \times 1)$ vector of environmental effects on each of the traits; $\Sigma_{j}$ denotes summation over all possible QTL positions on the chromosome; $\mathbf{v}_{\boldsymbol{j}}=$ the $(m \times 1)$ direction vector of the direction of the effects of the QTL alleles on different traits at position $j$; and $\mathrm{q}_{i j 1}\left(\mathrm{q}_{i j 2}\right)=$ the size of the QTL effect for the paternal (maternal) allele of animal $i$ at position $j$ along the direction $\mathbf{v}_{\boldsymbol{j}}$. For example, if $\left(\mathrm{q}_{i j 1}+\mathrm{q}_{i j 2}\right)=2$ and $\mathbf{v}=\left[\begin{array}{ll}1 & 2\end{array}\right]^{\prime}$ this gives an genotypic effect of 2 and 4 for traits 1 and 2, respectively. Another animal may have a QTL allele with a bigger (or smaller) effect, but the 1:2 ratio of the effects on traits 1 and 2 is the same for all animals at QTL position $j$. This restriction that the ratio of the allelic effects on each of the traits is constant across all the (infinite many) QTL alleles reduces the number of parameter estimates substantially [11]. 
Following Uimari and Sillanpaa [28] and Bink et al. [4], the dependencies between the effects of the fitted QTL are reduced by assuming that there is only one QTL per bracket. Furthermore, it will be assumed that there is little information to distinguish between QTL positions within a bracket, i.e. the likelihood is flat within a bracket. In order to reduce the number of possible QTL positions, only the midpoints of the brackets, $j$, are considered as putative QTL positions. Since the likelihood at every position within the bracket is assumed the same as that at the midpoint, combining the likelihood of a QTL at the midpoint with a prior probability at the midpoint of $P_{j}=$ sum of prior probabilities of having a QTL at each of the positions within the bracket, will yield the posterior probability of having a QTL within the bracket. Hence, by considering only the midpoints as putative QTL positions, the posterior probability of having a QTL at the midpoint yields an estimate of the posterior probability of having a QTL in the bracket. This probability of having a QTL within a bracket differs from the usual probability of having a QTL at a particular position, e.g. within a particular cM. The latter has consequences for the interpretation of the results, as will be discussed in the Discussion section.

\subsection{Joint posterior and likelihood}

In order to calculate the joint posterior probability density of the unknown parameters, the densities of all its components are written out first. The likelihood conditional on all unknowns is assumed multivariate normal:

$$
\mathrm{p}\left(\mathbf{y}_{i} \mid \mathbf{b}, \mathbf{u}_{i}, \mathbf{q}_{i . .}, \mathbf{v}_{.}, \mathbf{R}\right)=\mathrm{N}\left(\mathbf{y}_{i}-\mathbf{X}_{i} \mathbf{b}-\mathbf{u}_{i}-\Sigma_{j}\left(q_{i j 1}+q_{i j 2}\right) \mathbf{v}_{j} ; \mathbf{R}_{i}\right)
$$

where subscript . indicates all the possible values that this subscript can take, and $\mathbf{R}_{\boldsymbol{i}}=$ the $(m \times m)(\mathrm{co})$ variance matrix of the environmental effects, $\mathbf{e}_{\boldsymbol{i}}$. Here, the DYD's of sire $i$ are averaged over $\mathrm{d}_{i}$ daughters, such that $\mathbf{R}_{i}=\mathbf{R} / \mathrm{d}_{i}$.

The joint posterior probability density of the unknowns is:

$$
\mathrm{p}\left(\mathbf{b}, \mathbf{u}, \mathbf{q}_{\ldots}, \mathbf{v}_{.}, I_{.}, \mathbf{V}, \mathbf{G}, \mathbf{R} \mid \mathbf{y}, \mathbf{A}, \mathbf{H} .\right) \propto \Pi_{i}\left[\mathrm{p}\left(\mathbf{y}_{i} \mid \mathbf{b}, \mathbf{u}_{i}, \mathbf{q}_{i . .}, \mathbf{v}, \mathbf{R}\right)\right] \mathrm{p}(\mathbf{b}, \mathbf{u}, \mathbf{q}, \mathbf{v}, \mathbf{R})
$$

where $\mathrm{p}(\mathbf{b}, \mathbf{u}, \mathbf{q}, \mathbf{v}, \mathbf{R})$ is the joint prior distribution, which will be described in the next section.

\subsection{Components of the prior distribution}

The density of the polygenic effects, $\mathbf{u}_{i}$, is assumed multivariate normal:

$$
\mathrm{p}(\mathbf{u} \mid \mathbf{A}, \mathbf{G})=\mathrm{N}(0, \mathbf{A} \otimes \mathbf{G})
$$


where $\mathbf{u}=$ vector of polygenic effects where all $\mathbf{u}_{i}$ are stacked below each other; $\mathbf{A}=$ the additive relationship matrix which is calculated from the pedigree of the animals; and $\mathbf{G}=$ the $(m \times m)(\mathrm{co})$ variance matrix of the polygenic effects across the traits.

The density of the size of the QTL effects is assumed:

$$
\mathrm{p}\left(\mathbf{q} . j . \mid \mathbf{H}_{j}\right)=\mathrm{N}\left(0, \mathbf{H}_{j}\right)
$$

where $\mathbf{q}_{. j}$. $=$ vector of sizes of QTL effects at QTL position $j$, and $\mathbf{H}_{j}$ is the matrix of identical-by-descent (IBD) probabilities between the QTL alleles at position $j$, as indicated by the similarities between their marker haplotypes and includes the LD and linkage analysis information [20,21]. The $\mathbf{H}_{\boldsymbol{j}}$ matrix as defined here contains the IBD probabilities between all (founder and nonfounder) QTL alleles. Thus the $(i, k)$ element of $\mathbf{H}_{j}$ is the probability that the gametes $i$ and $k$ are IBD at QTL position $j$, based on the markers surrounding $j$. Note that, because a QTL is always IBD with itself, the diagonal elements of $\mathbf{H}_{j}$ are 1, i.e. sizes of QTL effects are standardized so that their variance is 1 . A bigger QTL is accommodated by having bigger $\mathbf{v}_{\boldsymbol{j}}$-values, whose distribution is shown below.

The estimation of the IBD probabilities at QTL position $j, \mathbf{H}_{j}$, is described in detail elsewhere $[20,21]$. Briefly, the IBD probability at the QTL between two base haplotypes (haplotypes of the first generation of genotyped animals) is based on the marker alleles that surround the QTL locus, i.e. many (non)identical marker alleles near the QTL imply a high (low) IBD probability at the QTL. This assumes that the haplotypes can be inferred from the genotyping of the base animals, which is only the case if the base animals have a large number of offspring (which is the case here). If linkage phases are unclear for a particular marker, the marker genotype is considered missing, which implies that this marker is not used in the comparisons of the marker alleles of two haplotypes. The actual level of the IBD probability depends on the population where the haplotypes are sampled from: the effective size of this population (here assumed to be $N_{e}=100$ ) and the number of generations since an arbitrary founder population (assumed to be $T=100$ ). The probability of coalescence between the current and this founder population is calculated given the marker alleles that both haplotypes (whose IBD probability is calculated) have in common. Simulation showed that the estimates of the QTL position are rather insensitive to the assumptions about $T$ and $N_{e}$ [19]. If the two haplotypes occur in animals with a known common ancestor, then the calculation of the IBD probability at the QTL is modified to account for this. The above IBD probabilities between base haplotypes account for the LD information in the 
marker data. The linkage analysis information is included by using the rules of Fernando and Grossman [14] to calculate the IBD probabilities between the base haplotypes and the haplotypes of their descendants and among the descendant's haplotypes.

Conditional on the variance of the direction vector, $\mathbf{v}_{j}$, and on whether position $j$ is included or excluded from the model, the direction vector is assumed multivariate normally distributed:

$$
\mathrm{p}\left(\mathbf{v}_{j} \mid \mathbf{V}, I_{j}\right)=\mathrm{N}\left(\mathbf{0}, I_{j}^{*} \mathbf{V}+\left(1-I_{j}\right)^{*} \mathbf{V} / 100\right)
$$

where $\mathbf{V}=(m \times m)$ diagonal matrix of variances of the elements of the direction vector, which represents the variability of the QTL effects across the positions where the QTL is fitted (since $\operatorname{Var}\left(\mathrm{q}_{i j k}=1\right)$ ); $I_{j}=$ an indicator variable, where $I_{j}=1$ indicates the presence of a QTL at position $j$ and $I_{j}=0$ indicates absence of a QTL at position $j$. Hence, if $I_{j}=0$, the variance of the direction vector is reduced by a factor 100 , which implies that the sampled $\mathbf{v}_{j}$ will be close to zero, and the QTL will be effectively removed from the model. This parameterization, where the QTL is effectively but not completely removed from the model when $I_{j}=0$, makes Gibbs-sampling of $I_{j}$ possible (as suggested by George and McCullogh [9]). The latter avoids using a Metropolis-Hastings sampling step in the MCMC algorithm. Note that, although the distribution of $\mathbf{v}_{\boldsymbol{j}}$ conditional on $\mathbf{V}$ and $I_{j}$ is Normal, because $\mathbf{V}$ and $I_{j}$ are themselves assumed to vary according to their prior distributions (see below), the unconditional distribution of $\mathbf{v}_{\boldsymbol{j}}$ is more thick-tailed than the Normal distributions and should thus be able to accommodate a wider range of values for the sizes of QTL effects.

The prior distribution of the indicator variable, $I_{j}$, is:

$$
\mathrm{p}\left(I_{j}\right)=\operatorname{Bernoulli}\left(P_{j}\right)
$$

where $P_{j}$ is the prior probability of having a QTL at midpoint $j . P_{j}$ was assumed 0.0163 times the number of $\mathrm{cM}$ that bracket $j$ was long, where the value of 0.0163 was based on the idea that previous analyses (e.g. [8]) already found a QTL in this region, i.e. there was a probability of 1 of having a QTL within the $61.24 \mathrm{cM}$ that was investigated $(1 / 61.24=0.0163)$.

The $i$ th diagonal element of $\mathbf{V}$, i.e. the variance of the $i$ th element of the direction vector $\mathbf{v}_{\boldsymbol{j}}$, had a (slightly informative) inverse chi-squared prior distribution with 10 degrees of freedom (some preliminary analysis with a flat prior for $\mathrm{V}_{i i}$ showed unreasonably large fluctuations for this parameter):

$$
\mathrm{p}\left(\mathrm{V}_{i i}\right)=\chi^{-2}\left(\mathrm{~S}_{0(i i)}, 10\right)
$$


where the scale parameter $\mathrm{S}_{0(i i)}$ was chosen such that the means of the $\chi^{-2}$ distribution equaled 409.2, 0.506, and 0.322, for milk, fat and protein yield, respectively. These means were based on the assumption and that the traits are affected by about 100 QTL, and single trait variance component analyses (without fitting a QTL), revealed sire variances of $40920,50.6$, and $32.2 \mathrm{~kg}^{2}$, respectively. A drawback of this empirical Bayes procedure, where hyperparameters are estimated first from the data, is that the data are used twice (first to estimate the hyperparameters and later to estimate the other parameters) and thus that the credible intervals of the parameter estimates will be underestimated.

The polygenic and residual (co)variance matrices, $\mathbf{G}$ and $\mathbf{R}$, were assumed to have an $m$-variate inverted Wishart distribution as a prior, which was parameterized such that the distribution was uniform for these matrices [27]:

$$
\mathrm{p}(\mathbf{G})=\operatorname{IW}_{m}\left(\mathbf{0}_{m \times m},-(m+1)\right), \quad \text { and } \mathrm{p}(\mathbf{R})=\operatorname{IW}_{m}\left(\mathbf{0}_{m \times m},-(m+1)\right)
$$

where $\mathbf{0}_{\boldsymbol{m} \times \boldsymbol{m}}=\mathrm{a}(m \times m)$ matrix of zeros, and the number of degrees of freedom was $-(m+1)=-4$, here. The fixed effects, $\mathbf{b}$, also had a uniform distribution as prior:

$$
\mathrm{p}(\mathbf{b}) \propto \text { constant. }
$$

The complete joint prior distribution now becomes:

$$
\mathrm{p}(\mathbf{b}, \mathbf{u}, \mathbf{q}, \mathbf{v}, \mathbf{R})=\mathrm{p}(\mathbf{u} \mid \mathbf{A}, \mathbf{G}) \mathrm{p}(\mathbf{V}) \mathrm{p}(\mathbf{R}) \mathrm{p}(\mathbf{G}) \mathrm{p}(\mathbf{b}) \Pi_{j} \mathrm{p}\left(\mathbf{q}_{. j} . \mathbf{H}_{j}\right) \mathrm{p}\left(\mathbf{v}_{j} \mid \mathbf{V}, I_{j}\right) \mathrm{p}\left(I_{j}\right) .
$$

Equations (2) and (3) together fully describe the joint posterior distribution, and Gibbs sampling was used to sample from this posterior distribution. The latter required fully conditional distributions for all the parameters involved, which are given in the Appendix.

\subsection{Data}

The basic data came from a grand-daughter design and have been described by Farnir et al. [8]. There were 1033 bulls with DYDs for milk, fat and protein yield. The bulls were distributed over 22 grand-sire families. The known pedigree of the 1033 bulls consisted of 3549 entries. The 1033 bulls and 22 grandsires were marker genotyped for 30 markers along chromosome 14 at positions $0,0.01,0.02,0.03,0.04,1.04,3.04,3.14,3.24,7.24,10.24,12.24$, $12.34,12.44,12.54,13.64,17.64,19.64,22.64,22.74,22.84,22.94,23.04$, $23.14,24.24,28.24,35.24,42.24,45.24,61.24 \mathrm{cM}$, where BULGE9 is the 
first marker at position 0 . Note that the first 5 markers are very close to each other. This results in 4 marker brackets of $0.01 \mathrm{cM}$ at the beginning of the chromosome segment. The second marker, which is a SNP marker, was identified by Grisart et al. [13] as the DGAT1 mutation.

\section{RESULTS}

Ten separate MCMC chains were run containing 200000 cycles each, of which the first 10000 cycles were discarded as burn-in. Due to the large amount of computer time involved, no alternative runs with different prior distributions were conducted, i.e. a sensitivity analysis to assess the effect of alternative prior distributions was not possible. Convergence was monitored by comparing the posterior probabilities of having a QTL at the putative QTL position across MCMC chains. This revealed that there was poor mixing at the beginning of the chromosome segment, i.e. a QTL was fitted in any of the first 4 brackets and hardly ever moved to another bracket (different chains fixed the QTL in a different bracket, out of the first four brackets). This suggested that there was a large QTL at the beginning of the chromosome, but that the MCMC analysis could not pinpoint the position of the QTL to one of the first 4 brackets. Figure 1 shows the posterior probabilities of $I_{j}=1$ averaged over the 10 chains. The posterior probabilities were obtained by calculating the fraction of Gibbs-cycles in which $I_{j}=1$. The posterior probability shows a sharp peak at the beginning of the chromosome segment, and very little evidence for a QTL at the remaining of the chromosome. The sharp peak at the beginning agrees approximately with the position of the DGAT1 mutation that was found by Grisart et al. [13], but not exactly. The DGAT1-SNP is at the end and beginning of brackets 1 and 2, respectively, and the posterior probability reached its highest point in bracket 3 . However, the differences in the posterior probability between brackets $1,2,3$, and 4 are small and may well be due to chance, since the QTL was fitted in 3 out of $10 \mathrm{MCMC}$ chains in bracket 3 (resulting in a posterior probability of $\pm 30 \%$ ) and in 2 out of 10 chains in each of the brackets 1, 2 and 4 (resulting posterior probabilities $\pm 20 \%$ for these brackets). Hence, the mapping method seemed unable to distinguish between brackets 1 , 2, 3, and 4, which span a $0.04 \mathrm{cM}$ region.

Figure 2 shows a multitrait single QTL maximum likelihood analysis using genetic model (1), but with only one QTL fitted (using the model of [21], except that a multitrait implementation of ASREML [10] was used). Also this analysis shows a sharp peak at the beginning of the chromosome, but the decline of the peak is markedly less steep. This is probably because if there is no 


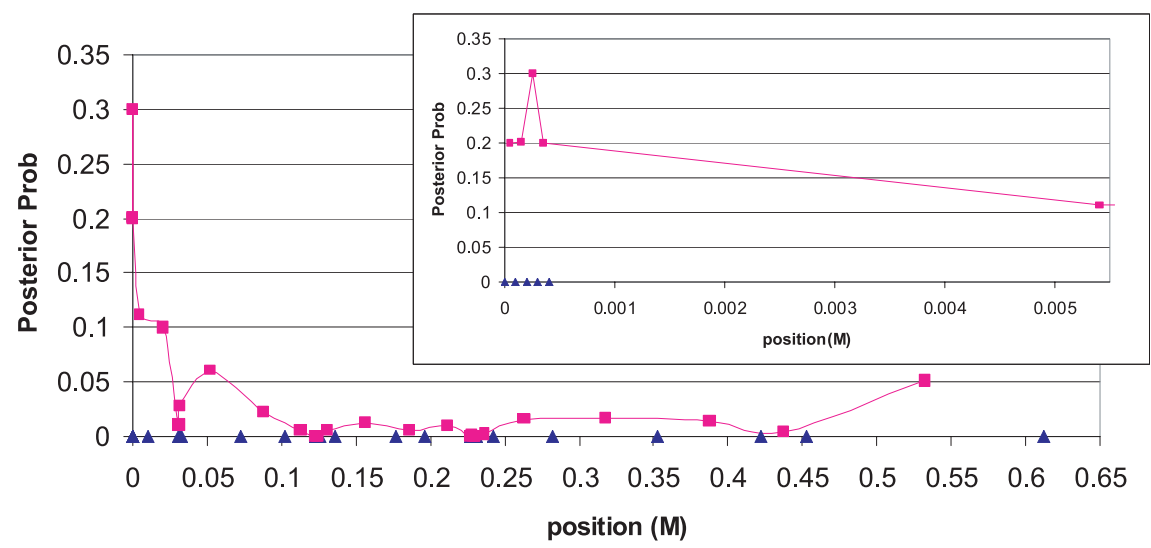

Figure 1. Multitrait-multi-QTL posterior probabilities of having a QTL affecting milk, fat and protein yield at each of the midpoints of the marker brackets ( $\Delta$ is marker position and is midpoint of the bracket). The insertion shows an enlargement of the first 5 marker brackets.

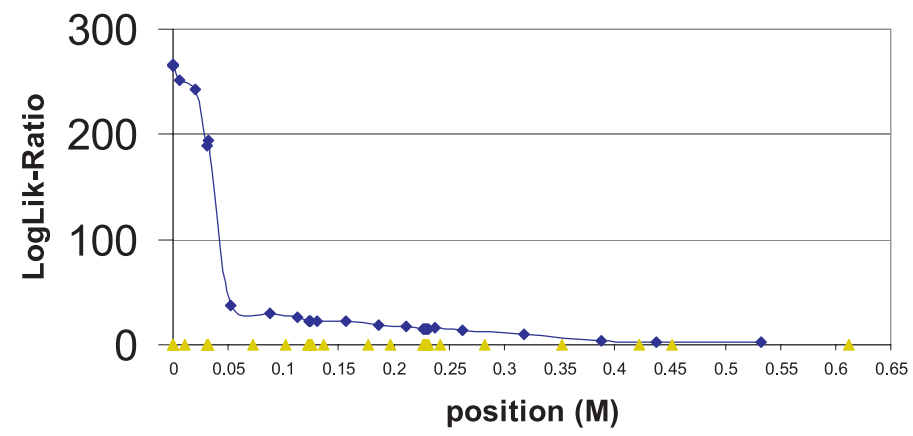

Figure 2. Multitrait-single-QTL loglikelihood ratio of having a QTL affecting milk, fat and protein yield versus no QTL affecting these traits plotted against the position of the QTL.

QTL fitted at the beginning of the chromosome, fitting a QTL some distance away from this large QTL will still pick up part of the effect of the QTL at the beginning of the chromosome.

The direction vector $\mathbf{v}_{3}$ was obtained by averaging over the Gibbs cycles where $I_{3}=1$. The estimate of this vector was $\mathbf{v}_{3}=[-50.6,1.78,-0.899]^{\prime}$, which agrees well with the direction of effects of the DGAT1 mutation found by Grisart et al. [13].

Figure 3 shows a histogram of the distribution of the sizes of the QTL alleles at locus 3, $\mathrm{q}_{i 3 k}$, where the effects were estimated from the cycles with $I_{3}=1$. The model assumes an infinite number of alleles, but if there 


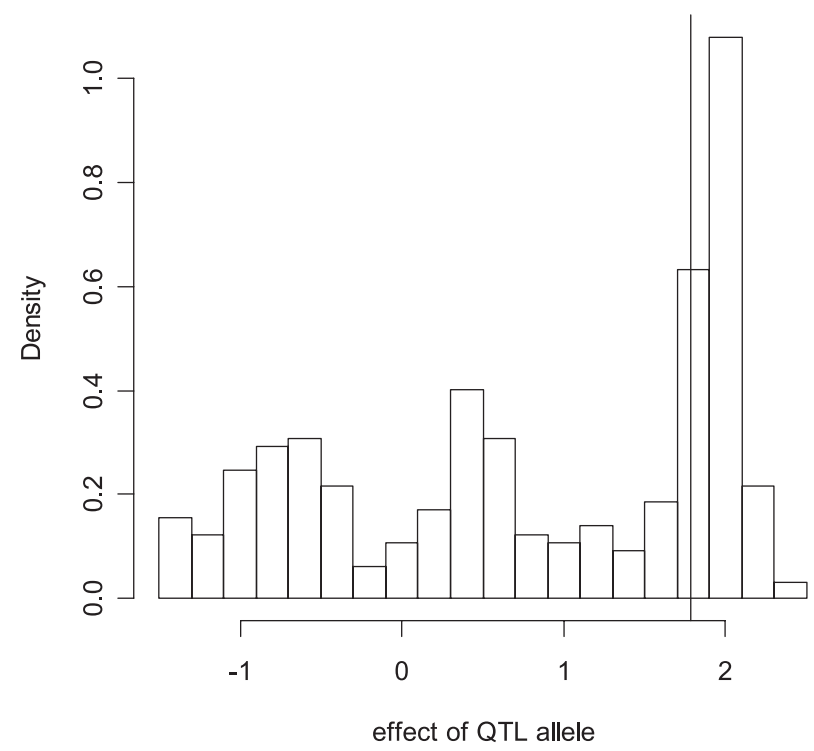

Figure 3. Histogram of the sizes of the effects of the $\mathrm{q}_{i 3 k}$-alleles in model (1). To the right of the vertical line are the 111 biggest $\mathrm{q}_{i 3 k}$-alleles that are associated with markerhaplotypes '2 11221 ' at the beginning of the chromosome (and other marker alleles further on the chromosome).

were only really two alleles segregating, many $\mathrm{q}_{i 3 k}$ would represent an estimate the same QTL allele effect and the distribution might be bimodal. In fact, the distributions seems to be tri-modal, indicating that perhaps some QTL alleles are showing the effect of the positive QTL allele, some that of the negative QTL allele, and some are less clearly associated with one of the QTL alleles. The $111 \mathrm{q}_{i 3 k}$-alleles with the biggest effects all occur on

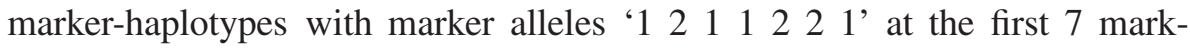
ers (and other alleles at later marker positions). The $32 \mathrm{q}_{i 3 k}$-alleles with the smallest effects occur all on marker-haplotypes with marker alleles '1 2221 ', at the markers 2,3,4 and 5, respectively (and other alleles at later marker positions). Apparently the haplotype ' 21112 ' for markers 2, 3, 4 and 5, is most strongly associated with the positive QTL allele and the haplotype

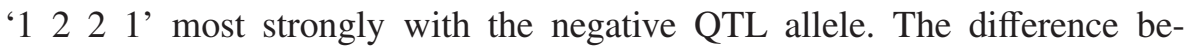

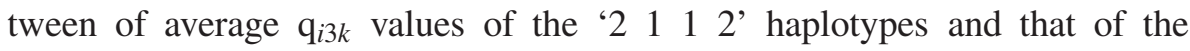
'1 2221 ' haplotypes is 3.09. Which makes our estimate of the effects of the QTL alleles on milk, fat and protein yield: $3.09^{*} \mathbf{v}_{3}=[-156.4,5.50,-2.78]$, which is well within the $95 \%$ confidence interval of the estimates of the DGAT1 mutation of Grisart et al. [13]. 


\section{DISCUSSION}

A multi-trait - multi-QTL mapping method was developed that used both the LD and linkage analysis information by extending the methods of Meuwissen and Goddard [19] and Meuwissen et al. [21]. The method was tested in practical data of chromosome 14, where Grisart et al. [13] recently discovered the DGAT1 mutation. The method mapped the DGAT1 gene to the first four marker brackets, which span a region of $0.04 \mathrm{cM}$. The reason why the current method could not map the DGAT1 - QTL as precisely as Grisart et al. is that they used additional information namely that the markers $1,3,4$, and 5, did not result in an amino acid substitution whereas marker 2 did, and that we only used information from Dutch bull families whereas the Grisart et al. data also contained sire families from New-Zealand.

The presented method is a variance component QTL mapping method, which implies that the infinite alleles model is used. In order to reduce the number of parameters, that need to be estimated, it was assumed that the effects of all these alleles were all along a single direction vector $\mathbf{v}_{j}$. If the QTL is truly bi-allelic, this assumption is valid because one group of the 'infinite' alleles are having a true effect of say [ $\left.\mathrm{v}_{1} \mathrm{v}_{2} \mathrm{v}_{3}\right]^{\prime}$ ' (the effect of QTL allele 1) and the remaining group of 'infinite' alleles are having a true effect of $\left[-\mathrm{v}_{1}-\mathrm{v}_{2}-\mathrm{v}_{3}\right]$ ' (the effect of QTL allele 2, when the average effect of the two QTL alleles is arbitrarily set to 0). Figure 4a gives an example of a biallelic QTL affecting 2 traits. If the QTL is however tri- or more allelic, there is no guarantee that the effects of the three groups of 'infinite' alleles are in the same direction. Figure $4 \mathrm{~b}$ gives an example of a 5-allelic QTL where the assumption of the QTL alleles having the same direction is true, while Figure $4 \mathrm{c}$ shows an example where this is not true. However, assuming that the 'infinite' alleles are all in the same direction may still yield a reasonable approximation because: (1) the three QTL alleles are three mutations in the same gene and may therefore have similar effects on the phenotype (although the extend/size of the effects may be different); and (2) more often than not, two of the QTL alleles will be much more frequent than the others and so dominate the data and make the two allele assumption approximately true. In their multi-trait QTL mapping method, Lund et al. [17] estimated the full (co)variance matrix of the QTL effects. It will be interesting to compare their results to ours, and see whether their methods finds correlations close 1 or -1 between the effects of the QTL on different traits. Also, the analysis of simulated data sets, where the true inheritance of one or several QTL is known, would be useful to reveal the properties of the presented method. 


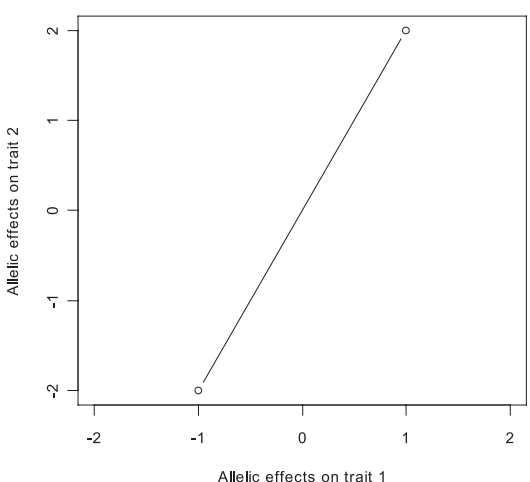

(a) a bi-allelic QTL

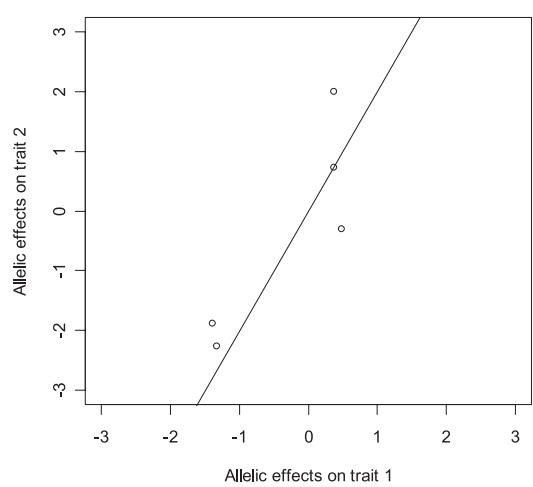

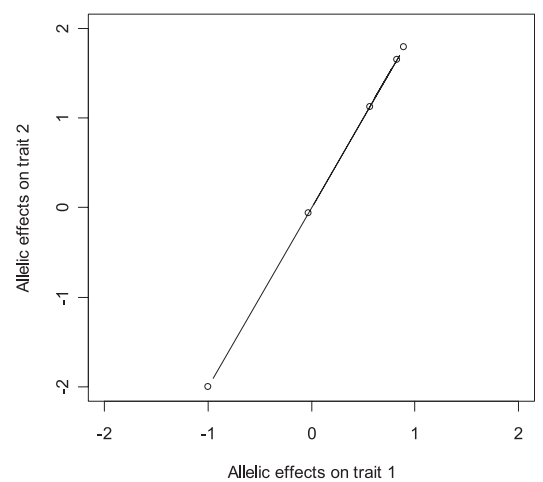

(b) 5-allelic QTL (direction of effects is the same for all alleles)

(c) 5-allelic QTL (correlation of 0.5 between allelic effects)

Figure 4. Examples of the effects of QTL alleles on two traits, for a bi-allelic QTL (a); for a 5-allelic QTL, but the direction of the effects is the same for all alleles (b); and for a 5-allelic QTL with a correlation of 0.5 between the allelic effects (c), on trait 1 and trait 2.

Only the midpoints of the brackets were considered as putative QTL positions here. This implies two assumptions: (1) there is only one QTL in the bracket (or having one QTL in a bracket is as likely as having 2 QTL with half the effect in the bracket); and (2) a QTL at the midpoint is as likely as anywhere else in the bracket. Both assumptions are approximately valid when bracket sizes are small because with small bracket sizes all positions within a bracket are very close to each other and thus give very similar results. Thus the presented method is mainly suited for fine mapping, where markers are close to each other. Since only a discrete set of QTL positions is considered, 
the present method should give similar results as grid-based QTL mapping methods where the number of QTL fitted is varied [3, 23, 25]. Alternatively, the model of Sillanpaa and Arjas [24] fits several QTL per marker bracket.

Another consequence of the above assumption is that the QTL at the midpoint absorbs all the likelihood of having one or more QTL at any of the positions within the brackets. Thus, when this likelihood is combined with the prior probability of having a QTL within the bracket, the resulting posterior probability refers to the posterior probability of having at least one QTL within the bracket. These posterior probabilities are thus particularly suited for choosing the bracket that most probably contains the QTL. A dip in the posterior probability in one of the brackets may thus be because the posterior probability per cM is reduced, or because the bracket size is reduced. An estimate of the posterior probability per cM may be obtained by simply dividing the posterior probability of the bracket by the bracket size (in $\mathrm{cM}$ ). However, the current posterior probability estimates reflect the probability of having one or more QTL, and especially for large brackets with high posterior probabilities (say $>0.8$ ), the posterior probability of having two QTL may be significant, and the latter is not accounted for when simply dividing posterior probability by bracket size. The latter estimate of the posterior probability per $\mathrm{cM}$ is thus biased downwards for large brackets with high posterior probabilities per bracket. For example, consider two brackets of 1 and $10 \mathrm{cM}$ with posterior probabilities of 0.1 and 1.00, respectively. Expressed per $\mathrm{cM}$ their posterior probabilities are both 0.1 , but for the $10 \mathrm{cM}$ bracket this is the maximum possible posterior probability, and thus this estimate may well be biased downwards (since an overprediction of the posterior probability in the $10 \mathrm{cM}$ bracket is not possible).

The method could be extended to include also the marker points as putative QTL positions. The combination of LD and LA information would be similar, although the computer code in the Appendix of [21] would need some adjustments to accommodate the marker positions. However, the LD information at the marker positions is very rigid: if the marker alleles differ, the IBD probability at the QTL position must be zero. This is not the case for a QTL that is close to the marker, especially when microsatellite markers are used, which are clearly not the causal mutation, and which mutate (i.e. change alleles) at a higher rate than the QTL position. On the other hand, the SNP marker positions 2, 3, 4, and 5, that might have caused the QTL effect could have been included as putative QTL positions, but the results would probably have been very similar because the midpoints are only $0.005 \mathrm{cM}$ away from the marker positions. 
The presented method used the infinite alleles model at the QTL, which makes it possible to combine LD and linkage analysis information, where the LD information is included as IBD probabilities between base population haplotypes (based on the information from flanking marker loci), and the linkage analysis information is included by using the markers to trace the inheritance of the QTL from base population animals to descendants (following [14]). An alternative approach for combining LD and linkage analysis information is to assume biallic QTL $[8,22,30,31]$. A complication with this approach is that many discretely distributed and highly interdependent parameters are needed, which need to be integrated out of the posterior distribution. The latter might result in the Gibbs sampler getting stuck in a subset of the parameter space. In the current approach, only the $I_{j}$ variable was discrete, and as the results indicated it could get stuck in the parameter space of $I_{j}=1$, when there was a very high probability of a QTL in this region. This could provide a problem, if there are also other brackets in the same region, i.e. when there are several very closely linked brackets (here: the distance between some midpoints was $0.01 \mathrm{cM})$. Running several Gibbs chains, where the chains got stuck in alternative brackets, revealed this problem. The latter was interpreted as that the QTL position could be in any of these closely linked brackets.

A consequence of the infinite alleles assumption is that it is difficult to include dominance effects into the model $[1,6]$, whereas their inclusion is easy for biallelic models. Since the additive effects of QTL are large, their dominance effect might also be large, and accounting for this might improve the precision of the QTL position estimate. Inclusion of dominance effects, however, results in many more equations per QTL position, and since there are many QTL positions in the present model, their inclusion would be computationally very demanding. Hence, dominance effects were not included here, and more research is needed towards a computationally efficient way of including these effects.

In the situation where single trait analyses of two traits revealed a QTL for both traits in (nearly) the same region, the question arises: is this result due to one QTL having pleiotropic effects on both traits, or are there two QTL each affecting one of the traits, i.e. the pleiotropy vs. close linkage question. The presented method could be used to answer this question, however, there are two difficulties: (1) a clear posterior probability peak at one of the bracket midpoints could still be due to two QTL in the same bracket (if the bracket size is small, this is a very difficult problem for any QTL mapping method); (2) if the model fits two QTL at two midpoints, it does not enforce that these two QTL have no pleiotropic effects, i.e. both QTL might have pleiotropic 
effects on both traits. The latter seems however a reasonable possibility. In fact, for related traits, e.g. height and weight of an animal, the assumption of no pleiotropic effects might be quite unrealistic (the animal would have to grow taller without becoming any heavier).

If we assume that there is only one QTL per bracket, the presented multiQTL analysis gives also an estimate of the number of QTL. However, this estimate is affected by the assumed prior distributions, mainly the prior probability of having a QTL $\operatorname{Pr}\left(I_{j}=1\right)$, and the prior distribution for the variances of sizes of QTL effects, i.e. does the model allow for many QTL with small effect or not. The use of prior distributions will increase the accuracy of the QTL estimation when they are informative and not misleading. Based on Farnir et al. [8] we assumed here a priori that that there was a QTL in the $61.24 \mathrm{cM}$ that was investigated. Further we assumed there were effectively about 100 QTL affecting the yield traits, which seems a reasonable number given the distribution of QTL effects [16]. This conservative prior (that most QTL are of small effect) did not prevent the analysis from estimating rather large effects for the QTL on chromosome 14 .

\section{ACKNOWLEDGEMENTS}

Holland Genetics, Livestock Improvement Cooperation, New Zealand, and Department of Genetics, Faculty of Veterinary Medicine, University of Liege, Belgium, are thanked for providing the data, and Holland Genetics is also acknowledged for financial support. An anonymous reviewer is thanked for many comments and suggestions for improvement.

\section{REFERENCES}

[1] Abney M., McPeek M.S., Ober C., Estimation of variance components of quantitative traits in inbred populations, Am. J. Hum. Genet. 66 (2000) 629-650.

[2] Akaike H., Information theory as an extension of the maximum likelihood principle, in: Petrov B.N., Csaki F. (Eds.), 2nd International Symposium on Information Theory, Akademiai Kiado, Budapest, 1973, pp. 267-281.

[3] Ball R.D., Bayesian methods for quantitative trait loci mapping based on model selection: approximate analysis using the Bayesian information criterion, Genetics 159 (2001) 1351-1364.

[4] Bink M.C.A.M., Uimari P., Sillanpää M.J., Janss L.L.G., Jansen R.C., Multiple QTL mapping in related plant populations via a pedigree-analysis approach, Theor. Appl. Genet. 104 (2002) 751-762.

[5] Broman K.W., Speed T.P., A model selection approach for identification of quantitative trait loci in experimental crosses, J. R. Stat. Soc. B 64 (2002) 641-656. 
[6] De Boer I.J.M., Hoeschele I., Genetic evaluation methods for populations with dominance and inbreeding, Theor. Appl. Genet. 86 (1993) 245-258.

[7] Farnir F., Coppieters W., Arranz J.-J., Berzi P., Cambisano N., Grisart B., Karim L., Marq F., Moreau L., Mni M., Nezer C., Simon P., Vanmanshoven P., Wagenaar D., Georges M., Extensive genome-wide linkage disequilibrium in cattle, Genome Res. 10 (2000) 220-227.

[8] Farnir F., Grisart B., Coppieters W., Riquet J., Berzi P., Cambisano N., Karim L., Mni M., Moisio S., Simon P., Wagenaar D., Vilkki J., Georges M., Simultaneous mining of linkage and linkage disequilibrium to fine map quantitative trait loci in outbred half-sib pedigrees: revisiting the location of a quantitative trait locus with major effect on milk production on bovine chromosome 14, Genetics 161 (2002) 275-287.

[9] George E.I., McCullogh R.E., Stochastic search variable selection, in: Gilks W.R., Richardson S., Spiegelhalter D.J. (Eds.), Markov chain Monte Carlo in Practice, Chapman and Hall, London, 1996, pp. 203-214.

[10] Gilmour A.R., Cullis B.R., Welham S.J., Thompson R., ASREML reference manual, 2000, ftp.res.bbsrc.ac.uk/pub/aar.

[11] Goddard M.E., The validity of genetic models underlying quantitative traits, Livest. Prod. Sci. 72 (2001) 117-127.

[12] Grignola F.E., Hoeschele I., Tier B., Mapping quantitative trait loci via residual maximum likelihood: I. Methodology, Genet. Sel. Evol. 28 (1996) 479-490.

[13] Grisart B., Coppieters W., Farnir F., Karim L., Ford C., Berzi P., Cambisano N., Mni M., Reid S., Simon P., Spelman R., Georges M., Snell R., Positional candidate cloning of a QTL in dairy cattle: Identification of a missense mutation in the bovine DGAT1 gene with major effect on milk yield and composition, Genome Res. 12 (2002) 222-231.

[14] Fernando R.L., Grossman M., Marker-assisted selection using best linear unbiased prediction, Genet. Sel. Evol. 21 (1989) 246-477.

[15] Hastbacka J., De La Chapelle A., Kaitila I., Sistonen P., Waever A., Lander E., Linkage disequilibrium mapping in isolated founder populations: diastrophic dysplasia in Finland, Nature Genet. 2 (1992) 204-211.

[16] Hayes B.J., Goddard M.E., The distribution of effects of genes affecting quantitative traits in livestock, Genet. Sel. Evol. 33 (2001) 209-229.

[17] Lund M., Sorensen P., Guldbrantsen B., Sorensen D.A., Multitrait fine mapping of quantitative trait loci using combined linkage disequilibrium and linkage analysis, Genetics 163 (2003) 405-410.

[18] Martinez O., Curnow R.N., Estimating the locations and the sizes of the effects of quantitative trait loci using flanking markers, Theor. Appl. Genet. 85 (1992) 480-488.

[19] Meuwissen T.H.E., Goddard M.E., Fine mapping of quantitative trait loci using linkage disequilibria with closely linked marker loci, Genetics 155 (2000) 421-430.

[20] Meuwissen T.H.E., Goddard M.E., Prediction of identity-by-descent probabilities from marker-haplotypes, Genet. Sel. Evol. 33 (2001) 605-634. 
[21] Meuwissen T.H.E., Karlsen A., Lien S., Olsaker I., Goddard M.E., Fine mapping of a quantitative trait locus for twinning rate using combined linkage and linkage disequilibrium mapping, Genetics 161 (2002) 373-379.

[22] Perez-Enciso M., Fine mapping of complex trait genes combining pedigree and linkage disequilibrium information: a Bayesian unified framework, Genetics 163 (2003) 1497-1510.

[23] Sen S., Churchill G.A., A statistical framework for quantitative trait mapping, Genetics 159 (2001) 371-387.

[24] Sillanpää M.J., Arjas E., Bayesian mapping of multiple quantitative trait loci from incomplete inbred line cross data, Genetics 148 (1998) 1373-1388.

[25] Sillanpää M.J., Kilpikari R., Ripatti S., Onkamo P., Uimari P., Bayesian association mapping for quantitative traits in a mixture of two populations, Genet. Epidem. 21 (Suppl. 1) (2001) S692-S699.

[26] Sillanpää M.J., Corander J., Model choice in gene mapping: what and why, Trends Genet. 18 (2002) 301-307.

[27] Sorensen D., Gianola D., Likelihood, Bayesian and MCMC methods in quantitative genetics, Springer-Verlag, New York, 2002.

[28] Uimari P., Sillanpää M.J., Bayesian oligogenic analysis of quantitative and qualitative traits in general pedigrees, Genet. Epidem. 21 (2001) 224-242.

[29] Weller J.I., Wiggans G.R., Vanraden P.M., Ron M., Application of a canonical transformation to detection of quantitative trait loci with the aid of genetic markers in a multi-trait experiment, Theor. Appl. Genet. 92 (1996) 998-1002.

[30] Wu R., Zeng Z.-B., Joint linkage and linkage disequilibrium mapping in natural populations, Genetics 157 (2001) 899-909.

[31] Wu R., Ma C.-X., Casella G., Joint linkage and linkage disequilibrium mapping of quantitative trait loci in natural populations, Genetics 160 (2002) 779-792.

\section{APPENDIX: THE FULLY CONDITIONAL DISTRIBUTIONS THAT WERE USED FOR THE GIBBS-SAMPLER}

The fully conditional distributions that are needed for Gibbs-sampling from posterior distribution (2) (see main text) are given here. More complete derivations of these fully condition distributions can be found in [27]. The symbols used correspond to those in the main text.

In each cycle of the Gibbs-chain, the fixed effects are sampled from:

$$
\mathbf{b} \mid \mathbf{u}, \mathbf{q}_{\ldots}, \mathbf{v}_{.}, I_{.}, \mathbf{V}, \mathbf{G}, \mathbf{R}, \mathbf{y} ., \mathbf{A}, \mathbf{H} \sim \mathrm{N}\left[\left(\mathbf{X}^{\prime} \mathbf{R}^{-1} \mathbf{X}\right)^{-1} \mathbf{X}^{\prime} \mathbf{R}^{-1} \mathbf{y}^{*} ;\left(\mathbf{X}^{\prime} \mathbf{R}^{-1} \mathbf{X}\right)^{-1}\right]
$$

where $\mathbf{y}^{*}$ denotes $\mathbf{y}$ corrected for all other effects except the fixed effects. The polygenic effects are sampled from (under the restriction that every animal has 
all records):

$\mathbf{u}_{i} \mid \mathbf{b}, \mathbf{u}_{-i}, \mathbf{q}_{\ldots}, \mathbf{v}_{.}, I_{.}, \mathbf{V}, \mathbf{G}, \mathbf{R}, \mathbf{y}_{.}, \mathbf{A}, \mathbf{H} \sim$

$$
\mathrm{N}\left[\left(\mathbf{R}^{-1}+\mathbf{G}^{-1} \mathrm{~A}^{i i}\right)^{-1}\left(\mathbf{R}^{-1} \mathbf{y}_{i}^{*}-\Sigma_{j \neq i} \mathrm{~A}^{i j} \mathbf{u}_{j}\right) ;\left(\mathbf{R}^{-1}+\mathbf{G}^{-1} \mathrm{~A}^{i i}\right)^{-1}\right],
$$

where $\mathbf{y}_{i}^{*}$ denotes $\mathbf{y}_{i}$ corrected for all other effects except for $\mathbf{u}$, and $\mathrm{A}^{i j}=$ the $(i, j)$ th element of the inverse of $\mathbf{A}$. Conditioning on $\mathbf{v}_{\boldsymbol{j}}$, which implies that $\mathbf{v}_{\boldsymbol{j}}$ can be considered as part of the design matrix for estimating the size of QTL effect $\left(\mathrm{q}_{i j k}\right)$, means that we can sample the size of the QTL effect at position $j$ from:

$\mathrm{q}_{i j k} \mid \mathbf{b}, \mathbf{u}, \mathbf{q}_{-(i j k)}, \mathbf{v}_{.}, I_{.}, \mathbf{V}, \mathbf{G}, \mathbf{R}, \mathbf{y}_{.}, \mathbf{A}, \mathbf{H} \sim$

$$
\begin{array}{r}
\mathrm{N}\left[\left(\mathbf{v}_{\boldsymbol{j}}^{\prime} \mathbf{R}^{-1} \mathbf{v}_{\boldsymbol{j}}+\mathrm{H}^{(i)(i)}\right)^{-1}\left(\mathbf{v}_{\boldsymbol{j}}^{\prime} \mathbf{R}^{-1} \mathbf{y}_{\boldsymbol{i}}^{*}-\Sigma_{l \neq(i)} \mathrm{H}^{(i) l} \mathrm{q}_{. j .\{l\}}-\mathbf{v}_{\boldsymbol{j}}^{\prime} \mathbf{R}^{-1} \mathbf{v}_{\boldsymbol{j}} \mathbf{q}_{i j \boldsymbol{k}^{\prime}}\right) ;\right. \\
\left.\left(\mathbf{v}_{\boldsymbol{j}}^{\prime} \mathbf{R}^{-1} \mathbf{v}_{\boldsymbol{j}}+\mathrm{H}^{(i)(i)}\right)^{-1}\right],
\end{array}
$$

where $\mathbf{y}_{i}^{*}$ denotes $\mathbf{y}_{\boldsymbol{i}}$ corrected for all other effects except for the QTL alleles at position $j,(i)$ denotes the row identification number of $\mathrm{q}_{i j k}$ in $\mathbf{H}^{-1} ; \mathrm{q}_{. j .\{l\}}$ denotes the QTL allele that belongs to row $l$ of the $\mathbf{H}^{-1} ; k^{\prime}=$ the maternal (paternal) allele if $k=1$ (if $k=2$ ).

The sampling of the direction vector $\mathbf{v}_{j}$ is by considering the model:

$$
\mathbf{y}^{*}=\mathbf{Q}_{j} \mathbf{v}_{j}+\mathrm{e}
$$

where $\mathbf{y}^{*}$ is again $\mathbf{y}$ corrected for all other effects except for the QTL alleles at position $j ; \mathbf{Q}_{j}$ is the $\left(n^{*} m \times m\right)$ design matrix for the direction vector, with elements $\mathrm{Q}_{j}(k, l)=\left(\mathrm{q}_{i j 1}+\mathrm{q}_{i j 2}\right)$ if the $k$ th element of $\mathbf{y}^{*}$ contains a record for trait $l$, otherwise: $\mathrm{Q}_{j}(k, l)=0$. Next the direction vector is sampled from:

$\mathbf{v}_{j} \mid \mathbf{b}, \mathbf{u}, \mathbf{q}_{\ldots}, \mathbf{v}_{-j}, I_{.}, \mathbf{V}, \mathbf{G}, \mathbf{R}, \mathbf{y}_{.}, \mathbf{A}, \mathbf{H} \sim$

$$
\mathrm{N}\left[\left(\mathbf{Q}_{j}^{\prime} \mathbf{R}^{-1} \mathbf{Q}_{j}+\mathbf{V}_{j}\right)^{-1} \mathbf{Q}_{j}^{\prime} \mathbf{R}^{-1} \mathbf{y}^{*} ;\left(\mathbf{Q}_{j}^{\prime} \mathbf{R}^{-1} \mathbf{Q}_{j}+\mathbf{V}_{j}\right)^{-1}\right]
$$

where $\mathbf{V}_{\boldsymbol{j}}=I_{j}^{*} \mathbf{V}+\left(1-I_{j}\right)^{*} \mathbf{V} / 100$ is a $(m \times m)$ diagonal matrix containing the variances of the direction vector $\mathbf{v}_{\boldsymbol{j}}$ at position $j$, and $\mathbf{V}=(m \times m)$ diagonal matrix of variances of the direction vectors for the QTL that have $I_{j}=1$, as explained in the main text. The fully conditional distribution of the $i$ th diagonal element of $\mathbf{V}$ was:

$$
\mathrm{V}_{i i} \mid \mathbf{v}_{.}, I \sim \chi^{-2}\left(\mathrm{~S}_{0(i i)}+\Sigma_{j} \mathrm{v}_{j(i)}^{2}\left(I_{j}+\left(1-I_{j}\right)^{*} 100\right), v+10\right)
$$


where $\mathrm{v}_{j(i)}=i$ th element of $\mathbf{v}_{j}$; the term $\left(I_{j}+\left(1-I_{j}\right)^{*} 100\right)$ takes value 1 if $I_{j}=1$, and value 100 (=1/[factor with which variance reduces when QTL is not fitted]) if $I_{j}=0 ; v=$ number of putative QTL positions that is considered.

The indicator variable, $I_{j}$, indicating whether a QTL is in or out of the model is sampled from:

$$
\begin{aligned}
I_{j} \mid \mathbf{v}_{\boldsymbol{j}}, \mathbf{V} \sim \operatorname{Bernoulli}\left[\phi\left(\mathbf{v}_{\boldsymbol{j}} ; \mathbf{0}, \mathbf{V}\right)^{*} \operatorname{Pr}\left(I_{j}=1\right) /\left\{\phi\left(\mathbf{v}_{\boldsymbol{j}} ; \mathbf{0}, \mathbf{V}\right)^{*} \operatorname{Pr}\left(I_{j}=1\right)\right.\right. \\
\left.\left.+\phi\left(\mathbf{v}_{\boldsymbol{j}} ; \mathbf{0}, \mathbf{V} / 100\right)^{*}\left(1-\operatorname{Pr}\left(I_{j}=1\right)\right)\right\}\right]
\end{aligned}
$$

where $\phi\left(\mathbf{v}_{\boldsymbol{j}} ; \mathbf{0}, \mathbf{V}\right)$ denotes the multivariate normal density function with mean $\mathbf{0}$ and variance-matrix $\mathbf{V}$.

The fully conditional distribution of the (co)variance matrices $\mathbf{G}$ and $\mathbf{R}$ was a $m$-variate inverted Wishart distribution with $n-m-1$ degrees of freedom [27]:

$$
\mathbf{G} \mid \mathbf{u}, \mathbf{A} \sim \operatorname{IW}_{m}\left(\mathbf{S}_{\mathrm{G}}, n-m-1\right), \quad \text { and } \mathbf{R} \mid \mathbf{e} . \sim \operatorname{IW}_{m}\left(\mathbf{S}_{\mathrm{R}}, n-m-1\right)
$$

where the $(k, l)$-element of the $(m \times m)$ matrix $\mathbf{S}_{\mathrm{G}}$ is $\mathrm{S}_{\mathrm{G}}(k, l)=\mathbf{u}_{(\boldsymbol{k})}^{\prime} \mathbf{A}^{-1} \mathbf{u}_{(l)}$ with $\mathbf{u}_{(\boldsymbol{k})}$ indicating the $(n \times 1)$ vector of polygenic effects for trait $k$; similarly the $(k, l)$ element of $\mathbf{S}_{\mathrm{R}}$ is $\mathrm{S}_{\mathrm{R}}(k, l)=\Sigma_{i} \mathrm{e}_{(i, k)} \mathrm{e}_{(i, l)} / \mathrm{d}_{i}$, where $\mathrm{e}_{(i, k)}=$ the environmental effect of animal $i$ for trait $k$, and $\mathrm{d}_{i}=$ the number of daughter records involved in the DYD of sire $i$.

To access this journal online: www.edpsciences.org 
